\title{
Clinical analysis of five case reports of ovarian teratoma with anti-N-methyl-D-aspartate receptor encephalitis
}

\author{
Chanyuan Li ${ }^{1,2}$, Fanliang Meng ${ }^{1}$, Shipeng Gong ${ }^{1}$ \\ ${ }^{1}$ Department of Obstetrics and Gynecology, Nanfang Hospital, Southern Medical University, Guangzhou, China; ${ }^{2}$ Department of Gynecology and \\ Obstetrics, The Fifth Affiliated Hospital of Sun Yat-sen University, Zhuhai, China \\ Correspondence to: Shipeng Gong; Fanliang Meng. Department of Obstetrics and Gynecology, Nanfang Hospital, Southern Medical University, \\ Guangzhou 510515, China. Email: gsp2103@163.com; 470693814@qq.com.
}

\begin{abstract}
We have collected the data of five patients with ovarian teratoma and anti-N-methyl-D-aspartate receptor (anti-NMDAR) encephalitis admitted to the Southern Hospital of Southern Medical University from June 2014 to December 2017, we found that all of them started with psychiatric symptoms, four had autonomic nervous system dysfunction, while all of them exposed to abnormal brain and pelvic imaging, four patients required mechanical ventilation to assist breathing during the hospital stay. The serum antiNMDAR antibodies were positive in five patients. After immunotherapy and surgery, all patients showed favorable prognosis. Pathology: four mature teratomas and one immature teratoma. In conclusion, patients with ovarian teratoma with anti-NMDAR encephalitis often begins with neurological and psychiatric symptoms, it is easy to be misdiagnosed due to the lack of overt symptoms, while the early detection and tumor resection combined with immunotherapy will win favorable prognosis.
\end{abstract}

Keywords: Ovarian teratoma; anti-N-methyl-D-aspartate receptor encephalitis (anti-NMDAR encephalitis); case reports

Submitted Dec 29, 2019. Accepted for publication Nov 23, 2020.

doi: 10.21037/apm-19-693

View this article at: http://dx.doi.org/10.21037/apm-19-693

\section{Introduction}

Marginal encephalitis is a rapidly developed paraneoplastic syndrome characterized by psychosis, seizures, sleeping disorders, hallucinations and short-term memory loss. In 2007, Tüzün found that some young female patients with benign teratoma had an unknown antigen, and was mainly expressed in the cell membrane of hippocampal neurons, which may be a new type of marginal paraneoplastic encephalitis (1). In the same year, Dalmau found that anti-N-methyl-D-aspartate receptor (anti-NMDAR) antibodies against hippocampal and prefrontal nerve cell membranes in such patients, and proposed the diagnosis of anti-NMDAR brains (2). Our study collected the clinical data of five patients with ovarian teratoma and anti-NMDAR encephalitis admitted to the Southern Hospital of Southern Medical University from June 2014 to December 2017, and analyzed their clinical symptoms, treatments and prognosis in order to gain experience for future clinical diagnosis and treatment, there is a summary of the clinical features in Table 1.

We present the following cases in accordance with the CARE reporting checklist (available at http://dx.doi. org/10.21037/apm-19-693).

\section{Case presentation}

\section{Case 1}

A 14 years old girl, admitted on December 20, 2017 due to being fever for 3 weeks, unusual behavior for 7 days, unconscious for 5 days. Examination: temperature $38-38.9^{\circ} \mathrm{C}$, imageology: a $6 \times 6 \mathrm{~mm}$ mixed mass in the left ovary, the anti-NMDAR antibody was positive both in the cerebrospinal fluid (CSF) and serum. Diagnosis: autoimmune encephalitis (anti-NMDAR encephalitis), ovarian tumor (left). Therapy: plasma exchange 2,000 mL for 5 days with two courses, gamma globulin $20 \mathrm{~g} / \mathrm{d}$ for 
Table 1 Summary of the patients' clinical features

\begin{tabular}{|c|c|c|c|c|c|c|c|}
\hline No. & CSF & Brain MRI & Pelvic image & EEG & Pathology & Tumor markers & $\begin{array}{l}\text { NMDAR } \\
\text { antibody }\end{array}$ \\
\hline 1 & Negative & $\begin{array}{l}\text { Sinus inflammation, } \\
\text { DWI signal change }\end{array}$ & $\begin{array}{l}\text { A } 22 \times 13 \times 38 \mathrm{~mm} \text { high-signal } \\
\text { cystic shadow in the right } \\
\text { ovary }\end{array}$ & Negative & $\begin{array}{l}\text { Mature } \\
\text { teratoma }\end{array}$ & Negative & Positive \\
\hline 2 & Negative & Sinus inflammation & $\begin{array}{l}\text { A mixed } 170 \times 190 \times 130 \mathrm{~mm} \\
\text { lesion in the right accessory }\end{array}$ & Negative & $\begin{array}{l}\text { Immature } \\
\text { teratoma }\end{array}$ & AFP $37.50 \mu \mathrm{g} / \mathrm{L}$ & Positive \\
\hline 4 & $\begin{array}{l}\text { Positive: } \\
\text { leukocytosis }\end{array}$ & Sinus inflammation & $\begin{array}{l}\text { A mixed } 10 \mathrm{~mm} \times 10 \mathrm{~mm} \\
\text { shadow on the right } \\
\text { accessory area }\end{array}$ & Negative & $\begin{array}{l}\text { Mature } \\
\text { teratoma }\end{array}$ & Negative & Positive \\
\hline
\end{tabular}

CSF, cerebrospinal fluid; MRI, magnetic resonance imaging; EEG, electroencephalograph; NMDAR, N-methyl-D-aspartate receptor.

5 days with two courses, methylprednisolone impact treatment ( $1 \mathrm{~g} 4$ days, $0.5 \mathrm{~g} 2$ days, $0.8 \mathrm{~g} 13$ days). Laparoscopic left adnexectomy was done on January 22, 2018. Intraoperative findings: the left ovary is pale and cordlike, about $20 \times 50 \mathrm{~mm}$ in size. Pathology: left ovarian mature cystic teratoma tumor. Discharge condition: the girl could be awakened and follow the word to act with occasional involuntary movement. The hospital stay was 68 days, 25 days in the intensive care unit, 22 days with the respiratory support. There was no tumor recurrence and symptom occur in the 6 months follow-up, which was assessed by imaging results and behavioral symptoms.

\section{Case 2}

A 15 years old girl, admitted to the hospital on July 29, 2017 due to the episodes of generalized convulsions and unusual behavior. Examination: lack of language competence, imageology: a $168 \times 33 \mathrm{~mm}$ mixed mass in the pelvic, antiNMDAR antibody was positive both in the CSF and serum. Diagnosis: autoimmune encephalitis (anti-NMDAR encephalitis), ovarian tumor. Therapy: we used valproate to stabilize her emotions, quetiapine and ziprasidone to improve mental symptoms, gamma globulin $25 \mathrm{~g} / \mathrm{d}$ for 5 days, methylprednisolone $1 \mathrm{~g}$ for 5 days treatment, the right adnexectomy was performed on August 14, 2017. Intraoperative findings: the right adnexal area was about $180 \times 130 \times 100 \mathrm{~mm}$ and lost its normal shape. Pathology: immature teratoma with visible hair follicles, glands, bone tissue and mature brain tissue inside. Discharge condition: the girl's self-care ability improved, without positive signs of nervous system. The hospital stay was 34 days, 30 days in the intensive care unit. There was no tumor recurrence and symptom occur 10 months after the operation, which was assessed by imaging results and behavioral symptoms.

\section{Case 3}

A 33 years old woman, due to acting unusual behavior for more than 20 days, aggravated convulsions for 2 weeks, admitted on September 6, 2017. Examination: occasional involuntary movements, imageology: a $108 \times 91 \times 75 \mathrm{~mm}$ mixed mass above the uterus in the pelvic, the anti-NMDAR antibody in CSF was positive. Diagnosis: autoimmune encephalitis (anti-NMDAR encephalitis, secondary epilepsy), ovarian tumor. Therapy: the patient was received gamma globulin $25 \mathrm{~g} / \mathrm{d}$ for 5 days, 2,000 $\mathrm{mL}$ plasma exchange for 5 times, and methylprednisolone $1 \mathrm{~g} / \mathrm{d}$ for 5 days shock treatment. The left ovary cystectomy was conducted on October 1, 2017, while intraoperative findings showed that the left ovary is enlarged to $80 \times 50 \times 60 \mathrm{~mm}$. Pathology: mature teratoma of the left ovary. Condition when discharge: she was in stable condition, could answer questions well except some intelligence problems. The hospital stay was 43 days, 3 days into the intensive care unit, 2 days with the respiratory support, and there was no tumor recurrence and symptom occur in the 8 months after the operation, which was assessed by imaging results and 
behavioral symptoms.

\section{Case 4}

A 21 years old woman, admitted on June 16, 2014 due to a headache with repeated fever for 17 days, unconsciousness for 6 days, aggravation with limb convulsions for 3 days. Examination: temperature $38-39{ }^{\circ} \mathrm{C}$, imageology: the circular low-density $10 \mathrm{~mm}$ mass in the right adnexal area combined with punctate calcification. Anti-NMDAR antibody was positive both in CSF and serum. Diagnosis: autoimmune encephalitis (anti-NMDAR encephalitis, secondary epilepsy), ovarian tumor. Therapy: treatment with the gamma globulin $20 \mathrm{~g} / \mathrm{d}$ for 5 days, 2,000 mL plasma exchange for 5 times, methylprednisolone $0.5 \mathrm{~g} / \mathrm{d}$ for 5 days impact treatment, the right oophorocystectomy was performed on July 14, 2014, we found that the right ovary was about $40 \times 30 \mathrm{~mm}$, with hair and adipose tissue inside. Pathology: right ovarian mature teratoma. Condition when discharge: the mental status was improved, accompanied by low fever without positive signs of nervous system. The hospital stay was 45 days, 40 days into the intensive care unit, 36 days with respiratory support. There was no tumor recurrence and symptom occur in 8 months after the operation, which was assessed by imaging results and behavioral symptoms.

\section{Case 5}

A 27 years old woman, admitted to the hospital on November 1, 2016 because of little words, fidgeting, laughing, and anorexia. The patient had a history of upper respiratory tract infection on October 26, 2016. Examination: anti-NMDAR antibodies in CSF and serum were positive, imageology: a $34 \times 17 \mathrm{~mm}$ weak liquid mass in the right ovary. Diagnosis: autoimmune encephalitis (anti-NMDAR encephalitis, symptomatic epilepsy), ovarian tumor. Therapy: she has been given a threeline treatment program: (November 5, 2016) first-line program: methylprednisolone $0.5 \mathrm{~g}$ shock treatment for 5 days, followed by $40 \mathrm{mg}$ methylprednisolone maintenance, plasma exchange for 5 days with 4 times, gamma globulin $0.4 \mathrm{~g} / \mathrm{kg} /$ day for 5 days with 3 times. (December 22, 2016) Second line protocol: cyclophosphamide $1.0 \mathrm{~g}$, rituximab $100 \mathrm{mg}$ every week for 4 consecutive weeks, one immunoadsorption treatment for 5 days. (December 12, 2017) Three-line program: gamma globulin $20 \mathrm{~g}$ combined with methylprednisolone $1 \mathrm{~g}$ shock treatment, gamma globulin for 5 days, methylprednisolone halved to $3 \mathrm{mg}$ every 3 days, methotrexate combined with dexamethasone was injected intrathecally 5 times. On November 5, 2016, the right oophorocystectomy was conducted. Pathology: right ovarian mature teratoma. While we found multiple hyperintense foci in pelvic through imageological examination on Aug 2017, then we performed the bilateral adnexectomy soon, the patient recovered well after the second surgery. Current condition: she is in a stable condition: acting low intensity activities, intaking semi-liquid food with coordinated swallowing function, answering the everyday problem fluently. She has been staying the hospital since November 1, 2016, staying in intensive care unit from November 6, 2016 to Aug 29, 2018, 557 days with the respiratory support. There was no tumor recurrence and symptom occur after the second operation in 10 months, which was assessed by imaging results and behavioral symptoms.

All procedures performed in studies involving human participants were in accordance with the ethical standards of the institutional and/or national research committee(s) and with the Helsinki Declaration (as revised in 2013). Written informed consents were obtained from all patients.

\section{Discussion}

Anti-NMDAR encephalitis is a marginal encephalitis associated with anti-NMDAR antibodies. NMDARs are composed of three subunits in the nervous tissues, and function as regulating synaptic transmission, triggering synaptic reorganization, and participating in learning and memory (3). In ovarian teratoma, there is a nerve tissue containing NMDAR subunit, which may be used as an antigenic substance to induce the production of antibody $\mathrm{IgG}$, which circulates in serum and CSF (4). All of the five patients were combined with teratoma, four cases were mature teratoma with one was immature teratoma, and one case was accompanied with endometriotic cyst, which also indicated that anti-NMDAR encephalitis may be associated with some ovarian tumors else.

Previous studies have divided the stage of the disease into five stages: the prodromal period, the psychiatric symptom period, the non-response period, the hyperkinetic period and the recovery period (5). In our part, five cases of patients with mental disorders and seizures, two patients with prodromal symptoms of upper respiratory tract infection, four patients need mechanical ventilation assisted breathing, indicating that the symptoms of NMDAR 


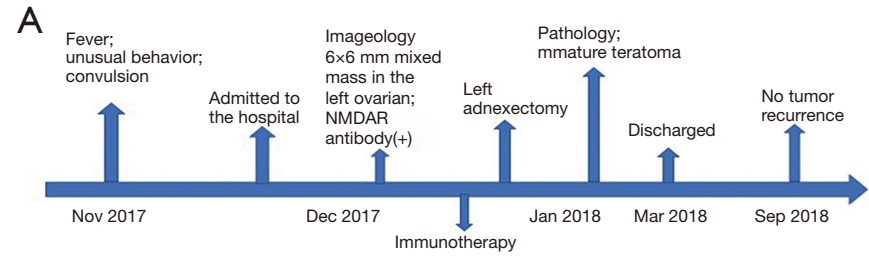

Timeline of case 1

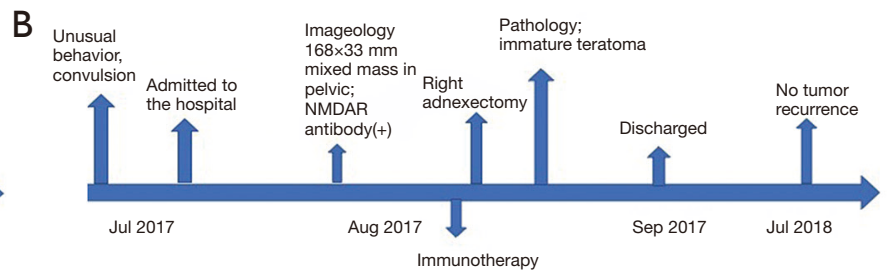

Timeline of case 2

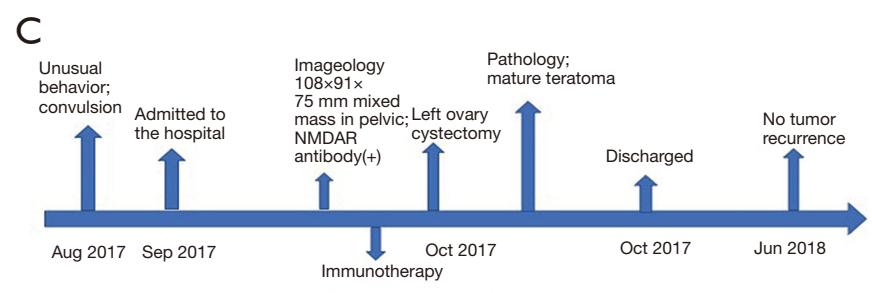

Timeline of case 3

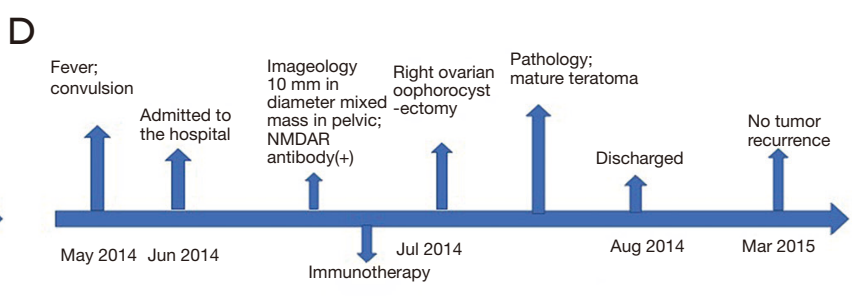

Timeline of case 4

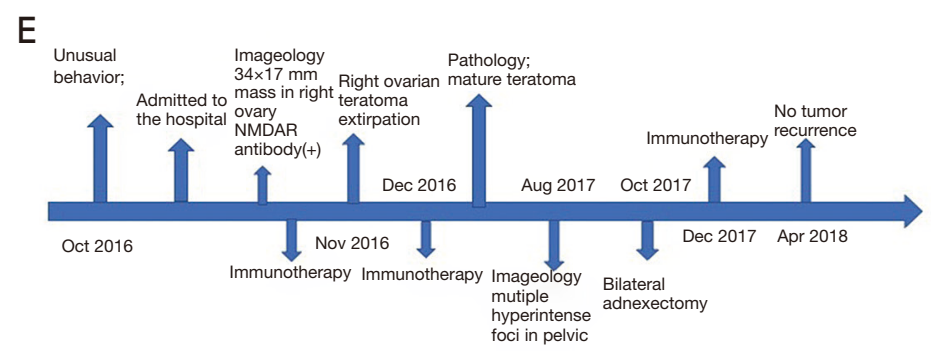

Timeline of case 5

Figure 1 Timeline of the treatment process. NMDAR, N-methyl-D-aspartate receptor.

encephalitis were variable and uncertain, leading to a higher rate of misdiagnosis.

Most patients can be diagnosed by typical symptoms such as psychiatric symptoms and positive anti-NMDAR antibody. It's reported that $40 \%$ of patients have abnormal head magnetic resonance imaging (MRI) results, while $85 \%$ of patients have abnormal electroencephalograph (EEG) results (5). The treatment of NMDAR encephalitis includes combination therapy: tumor resection and immunotherapy. Early operation is the key to cure the disease, the condition of the patients were improved obviously after combination therapy in our cases, and all of them were adhere to our therapeutic intervention, details of the treatments concluded in Figure 1.

In summary, anti-NMDAR encephalitis is a rare type of autoimmune disease, often combined with ovarian tumors, of which teratoma occurs most. Therefore, we should consider the possibility of this paraneoplastic disease in young women with psychiatric symptoms, then perform the combination therapy as soon as possible.

\section{Acknowledgments}

Funding: This study was funded by president foundation of Nanfang Hospital of Southern Medical University (2019B019).

\section{Footnote}

Reporting Checklist: The authors have completed the CARE reporting checklist. Available at http://dx.doi.org/10.21037/ apm-19-693

Conflicts of Interest: All authors have completed the ICMJE uniform disclosure form (available at http://dx.doi. org/10.21037/apm-19-693). The authors have no conflicts of interest to declare. 
Ethical Statement: The authors are accountable for all aspects of the work in ensuring that questions related to the accuracy or integrity of any part of the work are appropriately investigated and resolved. All procedures performed in studies involving human participants were in accordance with the ethical standards of the institutional and/or national research committee(s) and with the Helsinki Declaration (as revised in 2013). Written informed consents were obtained from all patients.

Open Access Statement: This is an Open Access article distributed in accordance with the Creative Commons Attribution-NonCommercial-NoDerivs 4.0 International License (CC BY-NC-ND 4.0), which permits the noncommercial replication and distribution of the article with the strict proviso that no changes or edits are made and the original work is properly cited (including links to both the formal publication through the relevant DOI and the license). See: https://creativecommons.org/licenses/by-nc-nd/4.0/.

Cite this article as: $\mathrm{Li} \mathrm{C}$, Meng F, Gong S. Clinical analysis of five case reports of ovarian teratoma with anti-N-methyl-Daspartate receptor encephalitis. Ann Palliat Med 2021;10(4):49504954. doi: 10.21037/apm-19-693

\section{References}

1. Tüzün E, Dalmau J. Limbic encephalitis and variants: classification, diagnosis and treatment. Neurologist 2007;13:261-71.

2. Dalmau J, Tüzün E, Wu HY, et al. Paraneoplastic anti-Nmethyl-D-aspartate receptor encephalitis associated with ovarian ter atoma. Ann Neurol 2007;61:25-36.

3. Dalmau J, Gleichman AJ, Hughes EG, et al. Anti-NMDAreceptor encephalitis: case series and analysis of the effects of antibodies. Lancet Neurol 2008;7:1091-8.

4. Dalmau J, Lancaster E, Martinez-Hernandez E, et al. Clinical experience and laboratory investigations in patients with anti-NMDAR encephalitis. Lancet Neurol 2011;10:63-74.

5. Iizuka T, Sakai F, Ide T, et al. Anti-NMDA receptor encephalitis in Japan: long-term outcome without tumor removal. Neurology 2008;70:504-11. 